\title{
Mayaro virus: a neglected threat could cause the next worldwide viral epidemic
}

\author{
Mânlio T de O Mota*,1, Clarita MS Avilla \& Maurício L Nogueira1 \\ ${ }^{1}$ Faculdade de Medicina de São José do Rio Preto (FAMERP), Av. Brigadeiro Faria Lima 5416, São Paulo, SP 15090-000, Brazil \\ *Author for correspondence: manliotasso@gmail.com
${ }^{\mathbf{6}}$ It is estimated that around $1 \%$ of all DENV-like cases in the northern region of South America could be caused by MAYV"

First draft submitted: 14 May 2019; Accepted for publication: 16 May 2019; Published online:

8 July 2019

Keywords: alphavirus $\bullet$ arbovirus $\bullet$ Mayaro fever $\bullet$ Mayaro virus $\bullet$ neglected disease $\bullet$ togavirus

In 2015, we published a review about Mayaro virus (MAYV), hoping to bring more attention to this important but neglected arbovirus [1]. Since then, even despite medical staff awareness being raised from several new cases in South and Central America and the Caribbean, the virus still stands largely neglected. The recent emergence of Chikungunya and Zika viruses (ZIKVs) highlights the importance of paying attention to less common arboviruses [2].

Arboviruses impose a serious burden to public health systems, mainly in tropical areas. It is estimated that around half of the world's population lives in areas at risk for dengue virus (DENV), probably the most-known of these viruses [3]. Global climate changes, rapid urbanization and higher mobility of the population increase the vector distribution of many of these diseases. Recently, mathematical models estimate that Aedes mosquitoes will infest major European and US cities in the coming decades [4]. The conditions that favor the spread of DENV vectors also favor the spread of other vectors and other arboviruses.

The awareness of politicians and medical staff about these diseases has increased in recent years; however, some arboviruses remain neglected. Many regions are at high risk in developing countries where resources are already limited, and thus, research is mainly directed to the most common arboviruses [1]. This creates a big gap in our knowledge and understanding of other important arboviruses, that may co-circulate in that country.

Despite the anthropogenic contributing factors, viral factors can also increase the circulation of a given virus. Viruses transmitted by vectors with a limited distribution can adapt to increase their transmission in more efficient vectors. Chikungunya virus (CHIKV) is an arthritogenic alphavirus and since its first recognized outbreak in 1952 in Tanzania [1], the virus has only caused limited outbreaks in Africa and Asia. CHIKV is maintained in Africa in a sylvatic zoonotic cycle involving canopy-dwelling Aedes species (Aedes africanus and Aedes furcifer-taylori) and wild primates, with humans only being incidentally infected [1]. In 2004, this situation changed drastically. A mutation in the $\mathrm{E} 1$ viral protein increased viral fitness of CHIKV, increasing replication efficiency in vectors and broadening its circulation. CHIKV caused large epidemics worldwide, with approximately 5 million cases over the last 15 years [5].

MAYV is a neglected arthritogenic alphavirus closely related to CHIKV. The disease it causes is very similar to that caused by CHIKV and other arthritogenic members of the Semliki Forest antigenic complex; a self-limited febrile illness usually presenting with highly incapacitating arthralgia that can persist for months or years [1].

It is believed that MAYV is maintained in nature in a sylvatic cycle, transmitted mainly by canopy dwelling Haemagogus mosquitoes, limited to forest areas and thus, only causing limited outbreaks. Human infections would only be spillover of this sylvatic transmission cycle, occurring only in rural and peri-urban areas [1]. However, it is very probable that urbanization could occur similarly to that of $\mathrm{CHIKV}$, adapting to replicate more efficiently in anthropophilic mosquitoes. The competence of the anthropophilic Aedes aegypti mosquitoes as a MAYV vector has already been demonstrated in laboratory [6] and wild populations [7]. Recently, in the Mato Grosso State in Brazil,

Future Medicine 
natural, vertical transmission of MAYV was reported in Aedes mosquitoes indicating that the virus is circulating in these mosquitoes [8].

Anopheles mosquitoes also show vector competence for MAYV transmission [9]. Moreover, MAYV was already isolated from Sabethes, Culex, Mansonia, Psorophora, Aedes serratus and Aedes scapularis [7], but most of this work was carried out years ago with little significance for current wild-type mosquito strains.

Recent epidemiological findings indicate that the virus already circulates in and around major urban centers in Brazil, such as Cuiabá (estimated population for 2018: 607,153) and Manaus (estimated population for 2018: $2,145,444)$ masked by concomitant outbreaks of dengue or other arboviruses [1,10-13]. The similarities of symptoms and vectors between MAYV and other arboviruses favor unnoticed circulation of MAYV in determined areas that contribute to underestimating the real situation of MAYV circulation. Furthermore, an important Mayaro outbreak occurred in the Goiás State, Brazil in 2016 [13]. This outbreak was largely neglected resulting from the ZIKV outbreak that occurred at the same time, but it raises the possibility that the virus may be migrating southwards in Brazil, following the steps of yellow fever virus in recent years [14].

The real number of MAYV cases is probably much higher than what is reported. It is estimated that around $1 \%$ of all DENV-like cases in the northern region of South America could be caused by MAYV [10]. In other words, MAYV circulation is much wider than previously believed and the virus is increasing its circulation. Epidemiological reports also indicate that MAYV could soon reach southern USA.

In 2015, a Mayaro fever case was identified in Haiti. An 8-year-old child from the rural area complained of fever and abdominal pain which was initially diagnosed as typhoid and treated with co-trimoxazole. Reverse Transcription-Polymerase Chain Reaction (RT-PCR) and inoculation in Vero 6 cells later detected DENV-1. However, the development of typical cytopathic effects for DENV-1 was much slower than DENV-1 positive plasma specimens from other patients, indicating that another virus may be present. A new RT-PCR using specific primers confirmed the presence of MAYV and the sequence of the amplicon revealed that the MAYV strain circulating in Haiti is different from that circulating in Peru, Bolivia, Venezuela, Trinidad and Tobago, and French Guiana, but very similar to samples isolated in Brazil [15].

This MAYV case indicates active circulation of MAYV in the Caribbean area, and thus, there is a potential threat of spread to southern USA as previously observed for other arboviruses such as CHIKV and ZIKV. Haiti has a large exchange of people with the USA, as well as similar vector species that may drive transmission of this virus to USA.

In addition to epidemiological reports, recent work showed that new viral strains can arise by recombination, a process that would broaden MAYV circulation through the potential increase in vector range and there is added risk of novel strains arising with higher pathogenicity. This work reported recent recombination events in the Amazon basin, in which a hybrid D/L genotype was identified [16]. However, it should be noted that the strain 2 BR14 (identified and sequenced by our group, accession number KT818520) never circulated in São Paulo, Brazil, but was an imported case from the Amazon region. Also a minor $\mathrm{N}$ (new) genotype, consisting of a single sequence, was isolated from strains of a large outbreak of Mayaro fever which occurred in Venezuela, in 2010 [17].

The Pan-American Health Organization (PAHO) recently reported, in an epidemiological alert, that Peru had 35 cases of Mayaro fever in 2018, and two cases so far in 2019 in the provinces of Quispicanchis (Cusco region) and La Mar (Ayacucho region). Ecuador has also reported five cases in 2019 [18].

These findings demonstrate the high plasticity of the MAYV genome indicating that the virus can increase its viral fitness, and consequently, could rapidly escalate circulation causing a worldwide epidemic, in a very similar way to CHIKV. The high urbanization potential of MAYV has brought attention to it, inducing some efforts to gain more understanding of the molecular mechanisms of MAYV pathogenesis in hopes of developing specific treatments, vaccines and detection methods [19-21]. However, there is no approved treatment or vaccine, to date. The main problem obstructing developments is low investments into research and flawed or even inexistent surveillance, which contributes to the increase of MAYV circulation. Much more attention must be paid to the MAYV in order to predict or prevent the spread of this virus. 


\section{Financial \& competing interests disclosure}

The authors acknowledge FAPESP (grants \#2014/05600-9 and \#2013/21719-3) and CNPq (ML Nogueira is a CNPq research fellow) for grants. The authors have no relevant affiliations or financial involvement with any organization or entity with a financial interest in or financial conflict with the subject matter or materials discussed in the manuscript.

No writing assistance was utilized in the production of this manuscript.

\section{References}

1. Mota MTDO, Ribeiro MR, Vedovello D, Nogueira ML. Mayaro virus: a neglected arbovirus of the Americas. Future Virol. 10(9), 1109-1122 (2015).

2. Zanotto PMA, Leite LCC. The challenges imposed by Dengue, Zika, and Chikungunya to Brazil. Front. Immunol. 9, 1964 (2018).

3. Maccormack-Gelles B, Lima Neto AS, Sousa GS et al. Epidemiological characteristics and determinants of Dengue transmission during epidemic and non-epidemic years in Fortaleza, Brazil: 2011-2015. PLoS Neglected Tropical Diseases 12(12), e0006990 (2018).

4. Liu-Helmersson J, Rocklov J, Sewe M, Brannstrom A. Climate change may enable Aedes aegypti infestation in major European cities by 2100. Environ. Res. 172, 693-699 (2019).

5. Pezzi L, Reusken CB, Weaver SC et al. GloPID-R report on Chikungunya, O’nyong-nyong and Mayaro virus, Part I: biological diagnostics. Antiviral Res. 166, 66-81 (2019).

6. Kantor AM, Lin J, Wang A, Thompson DC, Franz AWE. Infection pattern of Mayaro virus in Aedes aegypti (diptera: Culicidae) and transmission potential of the virus in mixed infections with Chikungunya virus. J. Med. Entomol. 56(3), 832-843 (2019).

7. Wiggins K, Eastmond B, Alto BW. Transmission potential of Mayaro virus in Florida Aedes aegypti and Aedes albopictus mosquitoes. Med. Vet. Entomol. 32(4), 436-442 (2018).

8. Maia LMS, Bezerra MCF, Costa MCS et al. Natural vertical infection by Dengue virus serotype 4, Zika virus and Mayaro virus in Aedes (stegomyia) aegypti and Aedes (stegomyia) albopictus. Med. Vet. Entomol. doi:10.1111/mve.12369 (2019) (Epub ahead of print).

9. Brustolin M, Pujhari S, Henderson CA, Rasgon JL. Anopheles mosquitoes may drive invasion and transmission of mayaro virus across geographically diverse regions. PLoS Negl. Trop. Dis. 12(11), e0006895 (2018).

10. Mota MTDO, Terzian AC, Silva MLCR, Estofolete C, Nogueira ML. Mosquito-transmitted viruses - the great Brazilian challenge. Braz. J. Microbiol. 47, 38-50 (2016).

11. Serra OP, Cardoso BF, Ribeiro ALM, Santos FaLD, Slhessarenko RD. Mayaro virus and Dengue virus 1 and 4 natural infection in culicids from Cuiabá, State of Mato Grosso, Brazil. Mem. Inst. Oswaldo Cruz 111, 20-29 (2016).

12. Vieira CJ, Silva DJ, Barreto ES et al. Detection of Mayaro virus infections during a Dengue outbreak in Mato Grosso, Brazil. Acta Trop. 147, 12-16 (2015).

13. Brunini S, Franca DDS, Silva JB et al. High frequency of Mayaro virus IgM among febrile patients, Central Brazil. Emerg. Infect. Dis. 23(6), 1025-1026 (2017).

14. Cunha MS, Da Costa AC, De Azevedo Fernandes NCC et al. Epizootics due to yellow fever virus in Sao Paulo State, Brazil: viral dissemination to new areas (2016-2017). Sci. Rep. 9(1), 5474 (2019).

15. Lednicky J, De Rochars VM, Elbadry M et al. Mayaro virus in child with acute febrile illness, Haiti, 2015. Emerg. Infect. Dis. 22(11), 2000-2002 (2016).

16. Mavian C, Rife BD, Dollar JJ et al. Emergence of recombinant Mayaro virus strains from the Amazon basin. Sci. Rep. 7(1), 8718 (2017).

17. Auguste AJ, Liria J, Forrester NL et al. Evolutionary and ecological characterization of Mayaro virus strains isolated during an outbreak, Venezuela, 2010. Emerg. Infect. Dis. 21(10), 1742-1750 (2015).

18. PAHO/WHO. Pan American Health Organization/World Health Organization. Epidemiological alert: Mayaro fever. 1 may 2019 (2019). www.paho.org/hq/index.php?option=com_content\&view=article\&id=15123:

1-may-2019-mayaro-fever-epidemiological-alert\&Itemid=42346\&lang=en

19. Choi H, Kudchodkar SB, Reuschel EL et al. Protective immunity by an engineered DNA vaccine for Mayaro virus. PLoS Negl. Trop. Dis. 13(2), e0007042 (2019).

20. Fumagalli MJ, De Souza WM, Romeiro MF, De Souza Costa MC, Slhessarenko RD, Figueiredo LTM. Development of an enzyme-linked immunosorbent assay to detect antibodies targeting recombinant envelope protein 2 of Mayaro virus. J. Clin. Microbiol. 57(5), pii:e01892-18 (2019).

21. Henss L, Yue C, Kandler J et al. Establishment of an alphavirus-specific neutralization assay to distinguish infections with different members of the Semliki forest complex. Viruses 11(1), 82 (2019). 
